\title{
GENERALIZED CONVEXITY OF THE INVERSE HYPERBOLIC COSINE FUNCTION
}

\author{
YUE HE AND GENDI WANG
}

Received 08 May, 2018

\begin{abstract}
The generalized convexity of the inverse hyperbolic cosine function related to the hyperbolic metric is investigated in this paper.
\end{abstract}

2010 Mathematics Subject Classification: 33B10; 26D07

Keywords: Hölder mean, convexity, concavity, inverse hyperbolic cosine function

\section{INTRODUCTION}

The hyperbolic functions and their inverses play an important role in the study of the hyperbolic geometry and quasiconformal mappings [1, 4, 5, 8, 9, 11, 12]. For example, the explicit formulas for the hyperbolic metric in the unit disk $\mathbb{B}^{2}$ and the upper half plane $\mathbb{H}^{2}$ are given in terms of the inverse hyperbolic sine and cosine functions, respectively, as follows [5, p.35, p.40]:

$$
\begin{gathered}
\rho_{\mathbb{B}^{2}}(x, y)=2 \operatorname{arsh} \frac{|x-y|}{\sqrt{\left(1-|x|^{2}\right)\left(1-|y|^{2}\right)}}, \\
\rho_{\mathbb{R}^{2}}(x, y)=\operatorname{arch}\left(1+\frac{|x-y|^{2}}{2 \operatorname{Im} x \operatorname{Im} y}\right) .
\end{gathered}
$$

In recent papers $[9,11]$, the authors investigated the properties of hyperbolic Lambert quadrilaterals in the unit disk by studying the inverse hyperbolic tangent and sine functions.

The study of the convexity/concavity with respect to Hölder means, or simply $H_{p, q}$-convexity/concavity, of special functions has attracted attentions of many researchers, see [2, 6, 7,9-11,13-17]. In particular, the convexity/concavity of the inverse hyperbolic tangent and sine functions has been studied in [9] and [11], respectively. For the definition of the above so-called generalized convexity/concavity, the reader is referred to Section 2.

In this paper, we continue the work of $[9,11]$ to study the generalized convexity for the inverse hyperbolic cosine function. Our main result is stated in the following theorem. 
Theorem 1. For $p, q \in \mathbb{R}$, the inverse hyperbolic cosine function arch is strictly $H_{p, q}$-convex on $(1,+\infty)$ if and only if $(p, q) \in D_{1}$, while arch is strictly $H_{p, q-}$ concave on $(1,+\infty)$ if and only if $(p, q) \in D_{2} \cup D_{3}$, where

$$
\begin{aligned}
& D_{1}=\{(p, q) \mid-\infty<p \leq 0,2 \leq q<+\infty\}, \\
& D_{2}=\left\{(p, q) \mid 0 \leq p \leq \frac{2}{3},-\infty<q \leq C(p)\right\}, \\
& D_{3}=\left\{(p, q) \mid \frac{2}{3}<p<+\infty,-\infty<q \leq 2\right\},
\end{aligned}
$$

and $C(p)$ is the same as in Lemma 3(4) with $C(0)=1$ and $C\left(\frac{2}{3}\right)=2$. In particular, for all $x, y \in(1,+\infty)$, there hold

$$
\operatorname{arch} \sqrt{x y} \leq \sqrt{\frac{\operatorname{arch}^{2} x+\operatorname{arch}^{2} y}{2}} \leq \operatorname{arch} \sqrt{\left(\frac{\sqrt[3]{x^{2}}+\sqrt[3]{y^{2}}}{2}\right)^{3}},
$$

with equalities if and only if $x=y$.

\section{PReliminaries}

For $r, s \in(0,+\infty)$, the Hölder mean of order $p$ is defined by

$$
H_{p}(r, s)=\left(\frac{r^{p}+s^{p}}{2}\right)^{\frac{1}{p}} \text { for } p \neq 0, \quad H_{0}(r, s)=\sqrt{r s} .
$$

For $p=1$, we get the arithmetic mean $A=H_{1}$; for $p=0$, the geometric mean $G=H_{0}$; and for $p=-1$, the harmonic mean $H=H_{-1}$. It is well known that $H_{p}(r, s)$ is continuous and increasing with respect to $p$.

A function $f: I \rightarrow J$ is called $H_{p, q}$-convex (concave) if it satisfies

$$
f\left(H_{p}(r, s)\right) \leq(\geq) H_{q}(f(r), f(s))
$$

for all $r, s \in I$, and strictly $H_{p, q}$-convex (concave) if the inequality is strict except for $r=s$.

The following monotone form of l'Hôpital's rule is of great use in deriving monotonicity properties and obtaining inequalities. See the extensive bibliography of [3].

Lemma 1 ([1, Theorem 1.25]). For $-\infty<a<b<\infty$, let functions $f, g:[a, b] \rightarrow$ $\mathbb{R}$ be continuous on $[a, b]$, and be differentiable on $(a, b)$. Let $g^{\prime}(x) \neq 0$ on $(a, b)$. If $f^{\prime}(x) / g^{\prime}(x)$ is increasing (deceasing) on $(a, b)$, then so are

$$
\frac{f(x)-f(a)}{g(x)-g(a)} \text { and } \frac{f(x)-f(b)}{g(x)-g(b)} .
$$

If $f^{\prime}(x) / g^{\prime}(x)$ is strictly monotone, then the monotonicity in the conclusion is also strict.

We prove the following three lemmas before giving the proof of Theorem 1. 
Lemma 2. Let $r \in(1,+\infty)$.

(1)The function $f_{1}(r) \equiv \frac{\operatorname{arch} r}{\sqrt{r^{2}-1}}$ is strictly decreasing with range $(0,1)$;

(2)The function $f_{2}(r) \equiv 2+\frac{\operatorname{arch} r+r \sqrt{r^{2}-1}-2 r^{3} \sqrt{r^{2}-1}}{\left(r^{2}-1\right)\left(\operatorname{arch} r+r \sqrt{r^{2}-1}\right)}$ is strictly decreasing with range $\left(0, \frac{2}{3}\right)$.

Proof. (1) Let $f_{11}(r)=\operatorname{arch} r$ and $f_{12}(r)=\sqrt{r^{2}-1}$, then $f_{11}\left(1^{+}\right)=f_{12}\left(1^{+}\right)=$ 0 . By differentiation, we have

$$
\frac{f_{11}^{\prime}(r)}{f_{12}^{\prime}(r)}=\frac{1}{r}
$$

which is strictly decreasing. Hence by Lemma 1 , the function $f_{1}$ is strictly decreasing with $f_{1}\left(1^{+}\right)=1$ and $f_{1}(+\infty)=0$.

(2) Let $f_{21}=\operatorname{arch} r+r \sqrt{r^{2}-1}-2 r^{3} \sqrt{r^{2}-1}$ and $f_{22}=\left(r^{2}-1\right)\left(\operatorname{arch} r+r \sqrt{r^{2}-1}\right)$, then $f_{21}\left(1^{+}\right)=f_{22}\left(1^{+}\right)=0$. By differentiation, we have

$$
\frac{f_{21}^{\prime}(r)}{f_{22}^{\prime}(r)}=\frac{-4}{2+\frac{f_{1}(r)}{r}}
$$

which is strictly decreasing by (1). Hence by Lemma 1 , the function $f_{2}$ is strictly decreasing with $f_{2}\left(1^{+}\right)=\frac{2}{3}$ and $f_{2}(+\infty)=0$.

Lemma 3. For $p \in \mathbb{R}$ and $r \in(1,+\infty)$, define

$$
h_{p}(r)=1+p \sqrt{r^{2}-1} \cdot \frac{\operatorname{arch} r}{r}+\frac{1}{\sqrt{r^{2}-1}} \cdot \frac{\operatorname{arch} r}{r} .
$$

(1) If $p \geq \frac{2}{3}$, then $h_{p}$ is strictly increasing with range $(2,+\infty)$.

(2) If $p<0$, then $h_{p}$ is strictly decreasing with range $(-\infty, 2)$.

(3) If $p=0$, then $h_{p}$ is strictly decreasing with range $(1,2)$.

(4) If $0<p<\frac{2}{3}$, then $h_{p}$ is not monotone and the range of $h_{p}$ is $[C(p),+\infty)$, where

$$
C(p)=\min _{r \in(1,+\infty)} h_{p}(r)
$$

with $1<C(p)<2$.

Proof. By Lemma 2(1), it is easy to get

$$
h_{p}\left(1^{+}\right)=2 \text { and } \quad h_{p}(+\infty)=\left\{\begin{array}{cc}
+\infty, & p>0 \\
1, & p=0 \\
-\infty, & p<0
\end{array}\right.
$$

By differentiation, we have

$$
h_{p}^{\prime}(r)=\frac{1}{r}\left(1+\frac{\operatorname{arch} r}{r \sqrt{r^{2}-1}}\right)\left(p-f_{2}(r)\right),
$$

where $f_{2}(r)$ is the same as in Lemma 2(2). 
By Lemma 2(2), we have (1)-(3).

(4) If $0<p<\frac{2}{3}$, since the range of $f_{2}$ is $\left(0, \frac{2}{3}\right)$, there exists one and only one point $r_{p} \in(1,+\infty)$ such that $p=f_{2}\left(r_{p}\right)$. Then $h_{p}$ is strictly decreasing on $\left(1, r_{p}\right)$ and increasing on $\left(r_{p},+\infty\right)$. Since $h_{p}$ is continuous in $r$, there exists

$$
C(p)=\min _{r \in(1,+\infty)} h_{p}(r)
$$

and $1<C(p)<2$.

Lemma 4. Let $p, q \in \mathbb{R}, r \in(1,+\infty)$, and $C(p)$ be the same as in Lemma 3(4). Let

$$
g_{p, q}(r)=\frac{\operatorname{arch}^{q-1} r}{r^{p-1} \sqrt{r^{2}-1}} .
$$

(1) If $p \geq \frac{2}{3}$, then $g_{p, q}$ is strictly decreasing for each $q \leq 2$, and $g_{p, q}$ is not monotone for any $q>2$.

(2) If $p<0$, then $g_{p, q}$ is strictly increasing for each $q \geq 2$, and $g_{p, q}$ is not monotone for any $q<2$.

(3) If $p=0$, then $g_{p, q}$ is strictly increasing for each $q \geq 2$, and $g_{p, q}$ is strictly decreasing for each $q \leq 1$, and $g_{p, q}$ is not monotone for any $1<q<2$.

(4) If $0<p<\frac{2}{3}$, then $g_{p, q}$ is strictly decreasing for each $q \leq C(p)$, and $g_{p, q}$ is not monotone for any $q>C(p)$.

Proof. By logarithmic differentiation in $r$, we have

$$
\frac{g_{p, q}^{\prime}(r)}{g_{p, q}(r)}=\frac{1}{\sqrt{r^{2}-1} \cdot \operatorname{arch} r}\left(q-h_{p}(r)\right)
$$

where $h_{p}(r)$ is the same as in Lemma 3. Hence the results immediately follow from Lemma 3.

\section{PROOF OF MAIN RESULT}

We are now in a position to prove Theorem 1.

Proof of Theorem 1. Without loss of generality, we may assume that $1<x \leq y<$ $+\infty$. Let $t=H_{p}(x, y)$, then $x \leq t \leq y$ and

$$
\frac{\partial t}{\partial x}=\frac{1}{2}\left(\frac{x}{t}\right)^{p-1}
$$

The proof is divided into the following four cases.

Case 1. $p \neq 0$ and $q \neq 0$.

Define

$$
F(x, y)=\operatorname{arch}^{q}\left(H_{p}(x, y)\right)-\frac{\operatorname{arch}^{q} x+\operatorname{arch}^{q} y}{2} .
$$


By differentiation, we have

$$
\frac{\partial F}{\partial x}=\frac{q}{2} x^{p-1}\left(\frac{\operatorname{arch}^{q-1} t}{t^{p-1} \sqrt{t^{2}-1}}-\frac{\operatorname{arch}^{q-1} x}{x^{p-1} \sqrt{x^{2}-1}}\right)=\frac{q}{2} x^{p-1}\left(g_{p, q}(t)-g_{p, q}(x)\right),
$$

where $g_{p, q}$ is defined in Lemma 4 .

Case 1.1 $p \geq \frac{2}{3}$ and $q \leq 2$.

By Lemma 4(1), the function $g_{p, q}$ is strictly decreasing on $(1,+\infty)$.

Case 1.1.1 If $q>0$, then $\frac{\partial F}{\partial x} \leq 0$. Hence $F(x, y)$ is strictly decreasing and $F(x, y) \geq F(y, y)=0$. Namely,

$$
\operatorname{arch}\left(H_{p}(x, y)\right) \geq\left(\frac{\operatorname{arch}^{q} x+\operatorname{arch}^{q} y}{2}\right)^{\frac{1}{q}}=H_{q}(\operatorname{arch} x, \operatorname{arch} y),
$$

with equality if and only if $x=y$.

Case 1.1.2 If $q<0$, then $\frac{\partial F}{\partial x} \geq 0$. Hence $F(x, y)$ is strictly increasing and $F(x, y) \leq$ $F(y, y)=0$. Namely,

$$
\operatorname{arch}\left(H_{p}(x, y)\right) \geq\left(\frac{\operatorname{arch}^{q} x+\operatorname{arch}^{q} y}{2}\right)^{\frac{1}{q}}=H_{q}(\operatorname{arch} x, \operatorname{arch} y),
$$

with equality if and only if $x=y$.

In conclusion, arch is strictly $H_{p, q}$-concave on the whole interval $(1,+\infty)$ for $(p, q) \in\left\{(p, q) \mid \frac{2}{3} \leq p<+\infty, 0<q \leq 2\right\} \cup\left\{(p, q) \mid \frac{2}{3} \leq p<+\infty, q<0\right\}$.

Case $1.2 p \geq \frac{2}{3}$ and $q>2$.

By Lemma 4(1), the function $g_{p, q}$ is not monotone on $(1,+\infty)$. With an argument similar to Case 1.1 , it is easy to see that arch is neither $H_{p, q}$-concave nor $H_{p, q}$-convex on the whole interval $(1,+\infty)$ for $(p, q) \in\left\{(p, q) \mid p \geq \frac{2}{3}, q>2\right\}$.

Case $1.3 p<0$ and $q \geq 2$.

By Lemma 4(2), the function $g_{p, q}$ is strictly increasing on $(1,+\infty)$ and hence $\frac{\partial F}{\partial x} \geq 0$. Then $F(x, y)$ is strictly increasing and $F(x, y) \leq F(y, y)=0$. Namely,

$$
\operatorname{arch}\left(H_{p}(x, y)\right) \leq\left(\frac{\operatorname{arch}^{q} x+\operatorname{arch}^{q} y}{2}\right)^{\frac{1}{q}}=H_{q}(\operatorname{arch} x, \operatorname{arch} y),
$$

with equality if and only if $x=y$.

In conclusion, arch is strictly $H_{p, q}$-convex on the whole interval $(1,+\infty)$ for $(p, q) \in\{(p, q) \mid p<0, q \geq 2\}$.

Case $1.4 p<0$ and $q<2$.

By Lemma 4(2), the function $g_{p, q}$ is not monotone on $(1,+\infty)$. With an argument similar to Case 1.3, it is easy to see that arch is neither $H_{p, q}$-concave nor $H_{p, q}$-convex on the whole interval $(1,+\infty)$ for $(p, q) \in\{(p, q) \mid p<0, q<0\} \cup\{(p, q) \mid p<0,0<$ $q<2\}$.

Case $1.50<p<\frac{2}{3}$ and $q \leq C(p)$. 
By Lemma 4(4), the function $g_{p, q}$ is strictly decreasing on $(1,+\infty)$.

Case 1.5.1 If $q>0$, then $\frac{\partial F}{\partial x} \leq 0$. Hence $F(x, y)$ is strictly decreasing and $F(x, y) \geq F(y, y)=0$. Namely,

$$
\operatorname{arch}\left(H_{p}(x, y)\right) \geq\left(\frac{\operatorname{arch}^{q} x+\operatorname{arch}^{q} y}{2}\right)^{\frac{1}{q}}=H_{q}(\operatorname{arch} x, \operatorname{arch} y),
$$

with equality if and only if $x=y$.

Case 1.5.2 If $q<0$, then $\frac{\partial F}{\partial x} \geq 0$. Hence $F(x, y)$ is strictly increasing and $F(x, y) \leq$ $F(y, y)=0$. Namely,

$$
\operatorname{arch}\left(H_{p}(x, y)\right) \geq\left(\frac{\operatorname{arch}^{q} x+\operatorname{arch}^{q} y}{2}\right)^{\frac{1}{q}}=H_{q}(\operatorname{arch} x, \operatorname{arch} y),
$$

with equality if and only if $x=y$.

In conclusion, arch is strictly $H_{p, q}$-concave on the whole interval $(1,+\infty)$ for $(p, q) \in\left\{(p, q) \mid 0<p<\frac{2}{3}, 0<q \leq C(p)\right\} \cup\left\{(p, q) \mid 0<p<\frac{2}{3}, q<0\right\}$.

Case 1.6 $0<p<\frac{2}{3}$ and $q>C(p)$.

By Lemma 4(4), the function $g_{p, q}$ is not monotone on $(1,+\infty)$. With an argument similar to Case 1.5 , it is easy to see that arch is neither $H_{p, q}$-concave nor $H_{p, q}$-convex on the whole interval $(1,+\infty)$ for $(p, q) \in\left\{(p, q) \mid 0<p<\frac{2}{3}, q>C(p)\right\}$.

Case 2. $p \neq 0$ and $q=0$.

Define

$$
F(x, y)=\frac{\operatorname{arch}^{2}\left(H_{p}(x, y)\right)}{\operatorname{arch} x \cdot \operatorname{arch} y} .
$$

By logarithmic differentiation, we obtain

$$
\frac{1}{F} \frac{\partial F}{\partial x}=x^{p-1}\left(g_{p, 0}(t)-g_{p, 0}(x)\right)
$$

where $g_{p, 0}$ is defined in Lemma 4 .

Case 2.1 $p \geq \frac{2}{3}$ and $q=0$.

By Lemma $4(1)$, the function $g_{p, q}$ is strictly decreasing on $(1,+\infty)$ and hence $\frac{\partial F}{\partial x} \leq 0$. Then $F(x, y)$ is strictly decreasing and $F(x, y) \geq F(y, y)=1$. Namely,

$$
\operatorname{arch}\left(H_{p}(x, y)\right) \geq \sqrt{\operatorname{arch} x \cdot \operatorname{arch} y}=H_{0}(\operatorname{arch} x, \operatorname{arch} y),
$$

with equality if and only if $x=y$.

In conclusion, arch is strictly $H_{p, q}$-concave on the whole interval $(1,+\infty)$ for $(p, q) \in\left\{(p, q) \mid p \geq \frac{2}{3}, q=0\right\}$.

Case $2.2 p<0$ and $q=0$.

By Lemma 4(2), the function $g_{p, q}$ is not monotone on $(1,+\infty)$. With an argument similar to Case 2.1, it is easy to see that arch is neither $H_{p, q}$-concave nor $H_{p, q}$-convex on the whole interval $(1,+\infty)$ for $(p, q) \in\{(p, q) \mid p<0, q=0)\}$.

Case $2.30<p<\frac{2}{3}$ and $q=0$. 
By Lemma 4(4), the function $g_{p, q}$ is strictly decreasing on $(1,+\infty)$ and hence $\frac{\partial F}{\partial x} \leq 0$. Then $F(x, y)$ is strictly decreasing and $F(x, y) \geq F(y, y)=1$. Namely,

$$
\operatorname{arch}\left(H_{p}(x, y)\right) \geq \sqrt{\operatorname{arch} x \cdot \operatorname{arch} y}=H_{0}(\operatorname{arch} x, \operatorname{arch} y),
$$

with equality if and only if $x=y$.

In conclusion, arch is strictly $H_{p, q}$-concave on the whole interval $(1,+\infty)$ for $(p, q) \in\left\{(p, q) \mid 0<p<\frac{2}{3}, q=0\right\}$.

Case 3. $p=0$ and $q \neq 0$.

Define

$$
F(x, y)=\operatorname{arch}^{q}(\sqrt{x y})-\frac{\operatorname{arch}^{q} x+\operatorname{arch}^{q} y}{2} .
$$

By differentiation, we obtain

$$
\frac{\partial F}{\partial x}=\frac{q}{2 x}\left(g_{0, q}(t)-g_{0, q}(x)\right),
$$

where $g_{0, q}$ is defined in Lemma 4 .

Case $3.1 p=0$ and $q \geq 2$.

By Lemma 4(3), the function $g_{p, q}$ is strictly increasing on $(1,+\infty)$ and hence $\frac{\partial F}{\partial x} \geq 0$. Then $F(x, y)$ is strictly increasing and $F(x, y) \leq F(y, y)=0$. Namely,

$$
\operatorname{arch}\left(H_{0}(x, y)\right) \leq\left(\frac{\operatorname{arch}^{q} x+\operatorname{arch}^{q} y}{2}\right)^{\frac{1}{q}}=H_{q}(\operatorname{arch} x, \operatorname{arch} y),
$$

with equality if and only if $x=y$.

In conclusion, arch is strictly $H_{p, q}$-convex on the whole interval $(1,+\infty)$ for $(p, q) \in\{(p, q) \mid p=0, q \geq 2\}$.

Case $3.2 p=0$ and $q \leq 1$.

By Lemma 4(3), the function $g_{p, q}$ is strictly decreasing on $(1,+\infty)$.

Case 3.2.1 If $0<q \leq 1$, then $\frac{\partial F}{\partial x} \leq 0$. Hence $F(x, y)$ is strictly decreasing and $F(x, y) \geq F(y, y)=0$. Namely,

$$
\operatorname{arch}\left(H_{0}(x, y)\right) \geq\left(\frac{\operatorname{arch}^{q} x+\operatorname{arch}^{q} y}{2}\right)^{\frac{1}{q}}=H_{q}(\operatorname{arch} x, \operatorname{arch} y),
$$

with equality if and only if $x=y$.

Case 3.2.2 If $q<0$, then $\frac{\partial F}{\partial x} \geq 0$. Hence $F(x, y)$ is strictly increasing and $F(x, y) \leq$ $F(y, y)=0$. Namely,

$$
\operatorname{arch}\left(H_{0}(x, y)\right) \geq\left(\frac{\operatorname{arch}^{q} x+\operatorname{arch}^{q} y}{2}\right)^{\frac{1}{q}}=H_{q}(\operatorname{arch} x, \operatorname{arch} y),
$$

with equality if and only if $x=y$.

In conclusion, arch is strictly $H_{p, q}$-concave on the whole interval $(1,+\infty)$ for $(p, q) \in\{(p, q) \mid p=0,0<q \leq 1\} \cup\{(p, q) \mid p=0, q<0\}$.

Case $3.3 p=0$ and $1<q<2$. 
By Lemma 4(3), the function $g_{p, q}$ is not monotone on $(1,+\infty)$. With an argument similar to Case 3.2, it is easy to see that arch is neither $H_{p, q}$-concave nor $H_{p, q}$-convex on the whole interval $(1,+\infty)$ for $(p, q) \in\{(p, q) \mid p=0,1<q<2)\}$.

Case 4. $p=0$ and $q=0$.

By Case 2.3, for all $x, y \in(1,+\infty)$, we have

$$
\operatorname{arch}\left(H_{p}(x, y)\right) \geq H_{0}(\operatorname{arch} x, \operatorname{arch} y) \quad \text { for } \quad 0<p<\frac{2}{3} .
$$

By the continuity of $H_{p}$ in $p$ and arch in $x$, we have

$$
\operatorname{arch}\left(H_{0}(x, y)\right) \geq H_{0}(\operatorname{arch} x, \operatorname{arch} y),
$$

with equality if and only if $x=y$.

In conclusion, arch is strictly $H_{0,0}$-concave on the whole interval $(1,+\infty)$.

By Case 1.1 and Case 3.1, arch is strictly $H_{\frac{2}{3}}$, -concave and strictly $H_{0,2}$-convex on $(1,+\infty)$. Therefore, the inequalities (1.1) hold with equalities if and only if $x=y$.

This completes the proof of Theorem 1.

Setting $p=1=q$ in Theorem 1 , we easily obtain the concavity of arch.

Corollary 1. The inverse hyperbolic cosine function arch is strictly concave on $(1,+\infty)$

\section{ACKNOWLEDGMENTS}

This research was supported by National Natural Science Foundation of China (NNSFC) under Grant No.11601485 and No.11771400, and Science Foundation of Zhejiang Sci-Tech University (ZSTU) under Grant No.16062023 -Y.

\section{REFERENCES}

[1] G. D. Anderson, M. K. Vamanamurthy, and M. Vuorinen, Conformal Invariants, Inequalities, and Quasiconformal Maps. New York: John Wiley \& Sons, 1997.

[2] G. D. Anderson, M. K. Vamanamurthy, and M. Vuorinen, "Generalized convexity and inequalities." J. Math. Anal. Math., vol. 335, no. 2, pp. 1294-1308, 2007, doi: 10.1016/j.jmaa.2007.02.016.

[3] G. D. Anderson, M. Vuorinen, and X.-H. Zhang, Topics in special functions III. Analytic Number Theory, Approximation Theory, and Special Functions. New York: Springer, $2014 . \quad$ doi: 10.1007/978-1-4939-0258-3.

[4] J. W. Anderson, Hyperbolic Geometry. London: Springer-Verlag, 2005.

[5] A. F. Beardon, The Geometry of Discrete Groups. New York: Springer-Verlag, 1995. doi: 10.1007/978-1-4612-1146-4.

[6] Y.-M. Chu, Y.-F. Qiu, and M.-K. Wang, "Hölder mean inequalities for the complete elliptic integrals." Integral Transforms Spec. Funct., vol. 23, no. 7, pp. 521-527, 2012, doi: 10.1080/10652469.2011.609482.

[7] Y.-M. Chu, M.-K. Wang, Y.-P. Jiang, and S.-L. Qiu, "Concavity of the complete elliptic integrals of the second kind with respect to Hölder means." J. Math. Anal. Math., vol. 395, no. 2, pp. 637-642, 2012, doi: 10.1016/j.jmaa.2012.05.083.

[8] M. Vuorinen, “Geometry of metrics.” J. Anal., vol. 18, pp. 399-424, 2010. 
[9] M. Vuorinen and G.-D. Wang, "Hyperbolic Lambert quadrilaterals and quasiconformal mappings." Ann. Acad. Sci. Fenn. Math., vol. 38, pp. 433-453, 2013, doi: 10.5186/aasfm.2013.3845.

[10] G.-D. Wang, "The inverse hyperbolic tangent function and Jacobian sine function." J. Math. Anal. Math., vol. 448, no. 1, pp. 498-505, 2017, doi: 10.1016/j.jmaa.2016.11.002.

[11] G.-D. Wang, “The adjacent sides of hyperbolic lambert quadrilaterals.” Bull. Malays. Math. Sci. Soc. , vol. 41, pp. 1995-2010, 2018, doi: 10.1007/s40840-016-0439-7.

[12] G.-D. Wang and M. Vuorinen, "The visual angle metric and quasiregular maps." Proc. Amer. Math. Soc., vol. 144, no. 11, pp. 4899-4912, 2016, doi: 10.1090/proc/13188.

[13] G.-D. Wang, X.-H. Zhang, and Y.-M. Chu, "Hölder concavity and inequalities for Jacobian elliptic functions." Integral Transforms Spec. Funct., vol. 23, no. 5, pp. 337-345, 2012, doi: 10.1080/10652469.2011.590482.

[14] M.-K. Wang, Y.-M. Chu, S.-L. Qiu, and Y.-P. Jiang, "Convexity of the complete elliptic integrals of the first kind with respect to Hölder means." J. Math. Anal. Appl., vol. 388, no. 2, pp. 1141-1146, 2012, doi: 10.1016/j.jmaa.2011.10.063.

[15] X.-H. Zhang, "Solution to a conjecture on the Legendre M-function with an application to the generalized modulus." J. Math. Anal. Appl., vol. 431, no. 2, pp. 1190-1196, 2015, doi: 10.1016/j.jmaa.2015.06.033.

[16] X.-H. Zhang, "Monotonicity and functional inequalities for the complete p-elliptic integrals." $J$. Math. Anal. Appl., vol. 453, no. 2, pp. 942-953, 2017, doi: 10.1016/j.jmaa.2017.04.025.

[17] X.-H. Zhang, "On the generalized modulus." Ramanujan J. , vol. 43, no. 2, pp. 405-413, 2017, doi: 10.1007/s11139-015-9746-0.

Authors' addresses

Yue He

School of Science, Zhejiang Sci-Tech University, Hangzhou 310018, China

E-mail address: yuehe_zstu@163.com

Gendi Wang

School of Science, Zhejiang Sci-Tech University, Hangzhou 310018, China

E-mail address: gendi .wang@zstu.edu.cn 